\title{
Staged Hybrid Treatment with Branched Endovascular Aneurysm Repair of a Thoracoabdominal Aortic Aneurysm in the Presence of a Total Infrarenal Aortoiliac Occlusion
}

\author{
Mateja Andic and Mario Lescan \\ Department of Thoracic and Cardiovascular Surgery, University Medical Centre Tübingen, Tübingen, Germany
}

\begin{abstract}
Aortoiliac occlusive disease may limit the use of branched endovascular aneurysm repair (BEVAR) of thoracoabdominal aneurysms (TAAAs). Thus, infrarenal aortoiliac occlusion may preclude the use of BEVAR. We present a case involving a 67-yearold patient with a fast-progressing TAAA (diameter: $70 \mathrm{~mm}$ ) and a concomitant total aortoiliac occlusion. A multi-staged treatment concept included the creation of the access and the distal landing zone for the consecutive endovascular procedures through an aorto-right femoral-left popliteal bypass. At six-week intervals, thoracic endovascular aortic repair for the creation of the proximal landing zone and a 4-vessel BEVAR were accomplished. At 36 months, a type 111 endoleak occurred due to the fracture of the bridging stent-graft to the celiac trunk and the superior mesenteric artery. It was successfully treated with VBX stent-grafts. This case illustrates the importance of a staged hybrid approach in the management of complex aortic pathologies with poor access and insufficient distal landing zone.
\end{abstract}

Key Words: Thoracoabdominal aortic aneurysm, Endovascular aneurysm repair, Peripheral artery disease, Endovascular procedures, Arterial occlusive diseases
Received August 24, 2021

Revised November 10, 2021

Accepted December 9, 2021

Published on December 31, 2021

\section{Corresponding author: Mario Lescan}

Department of Thoracic and Cardiovascular Surgery, University Medical Center Tübingen, Hoppe-Seyler Strasse 3, D-72076 Tübingen, Germany

Tel: 49-7071-29-85047

Fax: 49-7071-29-4047

E-mail: mario.lescan@med.uni-tuebingen.de https://orcid.org/0000-0002-3124-4994

Copyright (c) 2021 The Korean Society for Vascular Surgery

This is an Open Access article distributed under the terms of the Creative Commons Attribution Non-Commercial License (http://creativecommons.org/licenses/by-nc/4.0) which permits unrestricted non-commercial use, distribution, and reproduction in any medium, provided the original work is properly cited.

Cite this article; Vasc Specialist Int 2021. https://doi.org/10.5758/vsi.210064

\section{INTRODUCTION}

Thoracoabdominal aortic aneurysms (TAAAs) are increasingly treated with branched endovascular aneurysm repair (BEVAR) to reduce postoperative morbidity and mortality in old patients with severe comorbidities, who are unfit for open thoracoabdominal repair [1]. Small diameters of the access vessels with severe calcifications or tortuosity limit the use of BEVAR and may lead to access site complications including femoral vessel thrombosis, leg ischemia or iliac artery injuries [2]. To overcome the barriers imposed by insufficient access, hybrid repair solutions with iliac conduits may be considered [3]. However, a total aortoiliac occlusion with juxtarenal thrombosis is associated not only with the absence of access vessels but also of the distal landing zone. Moreover, bilateral hypogastric occlusion and lumbar artery thrombosis coincide with a high risk of paraplegia in endovascular treatment lengths $>200 \mathrm{~mm}$ [4-6]. We outline an alternative treatment strategy to the open repair in a high-risk patient with an unusual pathologic constellation: total infrarenal aortoiliac occlusion in the presence of a fast-growing TAAA (Fig. 1).

The study was approved by the Independent Research Ethics Committee of the University of Tübingen (no. 626/2021A). Written informed consent was obtained from the patient for the publication of the case and the image material before the submission. 


\section{CASE}

An immobile 67-year-old male patient was presented to our center in a reduced general condition with severe peripheral arterial disease and rest pain in both legs. The comorbidities included coronary artery disease, chronic obstructive lung disease, nicotine abuse, and hypertension. Computed tomography angiography (CTA) showed total occlusion of infrarenal aorta, bilateral iliac and hypogastric arteries and additional thrombosis of the left common and superficial femoral artery (Fig. 2A). Moreover, a TAAA (diameter progress from $45 \mathrm{~mm}$ to $70 \mathrm{~mm}$ within 11 months) overriding the aortic occlusion was observed. Being unfit for open thoracoabdominal surgery, the patient was scheduled for urgent open repair of the infrarenal aortic occlusion and subsequent endovascular staged treatment of the TAAA.

In the first stage, we replaced the occluded infrarenal aorta with a bifurcated Dacron graft $(22 \mathrm{~mm} \times 11 \mathrm{~mm}$; Aesculap, Tuttlingen, Germany) after the median laparotomy and infrarenal aortic cross-clamping. The proximal end-toend anastomosis and the distal anastomoses to the common femoral artery on the right side and to the above-knee popliteal artery on the left side were sewn. An additional incision in the left groin was performed to accomplish an endto-end anastomosis of the Dacron graft to an 8-mm ePTFE graft (Gore, Flagstaff, AZ, USA) to obtain a sufficient graft length in order to revascularize the popliteal artery. On the ninth postoperative day, the patient was discharged from the hospital.

Six weeks later, a thoracic endovascular aortic repair procedure was performed to create a proximal landing zone for the BEVAR. The cerebrospinal fluid (CSF) drain was placed preoperatively to prevent spinal cord ischemia and a femoral cut-down to the Dacron graft on the left side was performed at both endovascular stages under general anes-
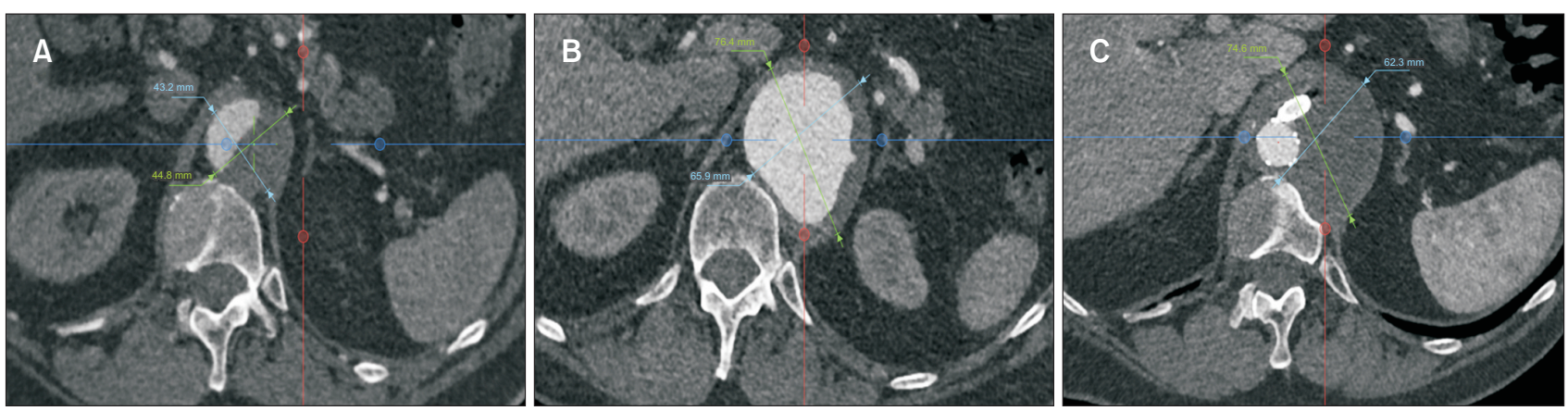

Fig. 1. Axial computed tomography scans (vertebra Th12) showing the diameter progress of the thoracoabdominal aneurysm at diagnosis (A) and 14 months thereafter (B). (C) Stable aortic diameter 22 months after the treatment completion.
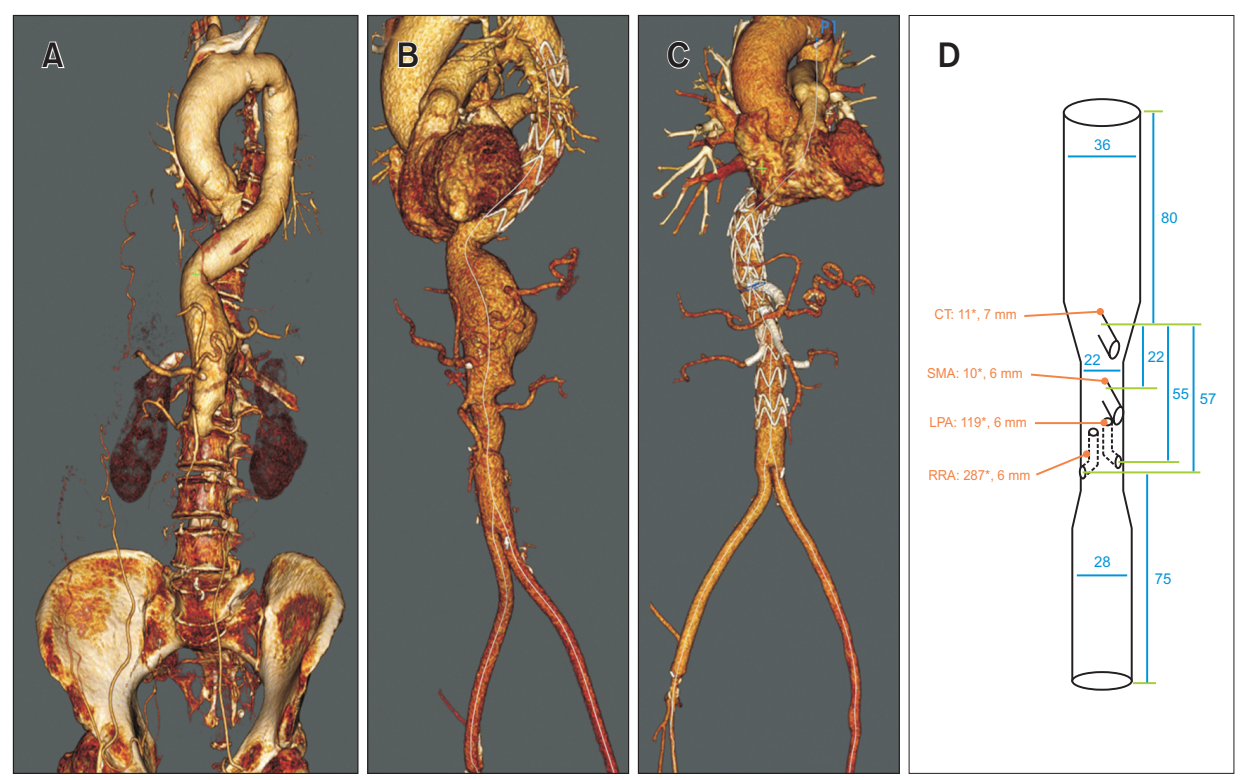

Fig. 2. Three-dimensional computed tomography volume rendering with thoracoabdominal aneurysm and infrarenal occlusion at admission $(A)$, after the replacement of the infrarenal aorta and thoracic endovascular aortic repair (B), and 22 months after branched endovascular aneurysm repair (C). (D) Form and dimensions of the custommade endograft with 2 directional branches for the superior mesenteric artery (SMA) and the celiac trunk (CT) and two inner branches for the renal arteries. LRA, left renal artery; RRA, right renal artery. 

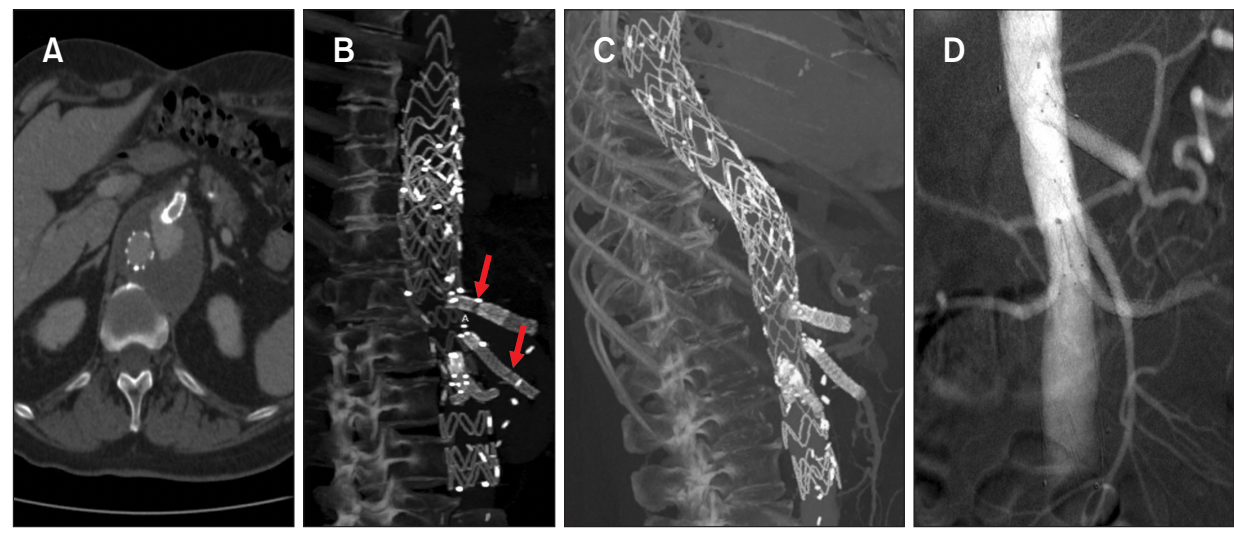

Fig. 3. Computed tomography (CT) scan showed type III endoleaks (A) from the fractures of bridging stent-grafts to the celiac axis and superior mesenteric artery (B; red arrows). After VBX implantation the CT scan showed fixed bridging stent-grafts (C) without endoleak in the completion angiography (D).

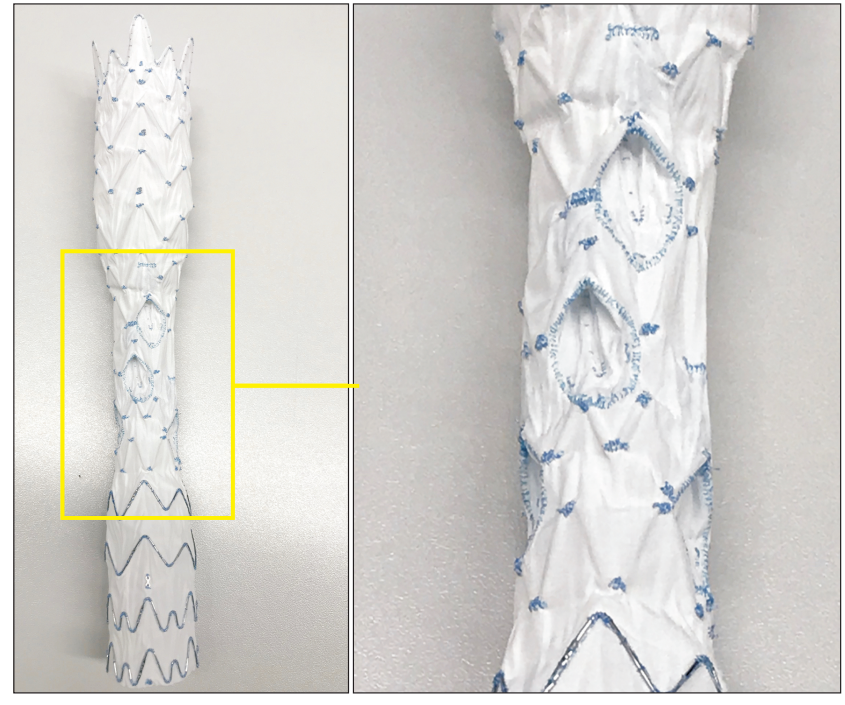

Fig. 4. Inner branch based and precannulated, off-the-shelf En-side stent-graft.

thesia. A Jotec 38/130/30-mm tapered thoracic endograft (Evita 3G; Jotec, Hechingen, Germany) was deployed in the descending aorta, proximally to the origin of the celiac axis (CA; Fig. 2B). The postoperative course was uneventful. Five days after the surgery, CT control scan was performed, which is standard practice in our clinic. The patient was discharged on the same day. Six weeks later, a custom-made endograft (Jotec Extra Design; Jotec) with two directional branches for the CA and superior mesenteric artery (SMA) and two inner branches for the renal arteries were implanted. The CSF drain was placed prior to procedure. Bridging stent-grafts (E-ventus; Jotec) were deployed through the left brachial artery cut-down (Fig. 2C, D). No technical complications developed during the postoperative course. The patient was discharged eight days later after recovering form postoperative delirium and reduced general condition after three complex surgeries in a relatively short period. However, the CTA showed no abnormality. Uneventful follow-ups were performed at 3, 6, 12, and 22 months after discharge with stable aneurysm diameters and completely excluded aneurysm without endoleaks. All endografts were patent and no re-interventions were required. At 36 months, a new type 111 endoleak was diagnosed due to the fracture of the bridging stent-grafts to the CA and SMA, which resulted in an aneurysm growth of $9 \mathrm{~mm}$ (Fig. 3A, B). The endoleak was fixed by VBX endografts (Gore) through transbrachial approach (Fig. 3C, D). The follow-up was uneventful 13 months after the bridging stent-graft correction.

\section{DISCUSSION}

Calcified or occluded access vessels limit the use of transfemoral BEVAR. Complementary implementation of open surgery to gain access has been previously described, however not to this extent as presented in this case report [7]. We demonstrated the maximum variant of access and distal landing zone creation in the form of an infrarenal aortic replacement in a patient with infrarenal aortic occlusion, who otherwise could not have been treated endovascularly and who was not a candidate for open surgery due to serious comorbidities.

Two technical notes need to be addressed. The first is the end-to-end proximal anastomosis instead of the endto-side anastomosis - which is usually performed for the treatment of infrarenal aortic occlusion - to avoid the incomplete stent-graft apposition in the distal landing zone and the risk of type $\mathrm{lb}$ endoleak. The straight distal landing zone of $30 \mathrm{~mm}$ in the Dacron graft ensured safe infrarenal sealing for the BEVAR. The second technical issue includes the narrow aortic anatomy at the level of renal arteries. The use of a custom-made endograft with 2 outer branches (CA, SMA) and two inner branches for the renal arteries allowed for a safe deployment of the main graft and the eased cannulation of the renal arteries. Alternative approaches in narrow aortic anatomies include the use of two custom- 
made fenestrations for the renal arteries instead of inner branches, or in case of urgencies surgeon-modified stentgrafts or chimneys [8-10]. The first precannulated off-theshelf BEVAR stent-graft based on inner branches (En-side; JOTEC Cryolife, Hechingen, Germany) has been released in 2020 in Europe. Since then, our endovascular TAAA strategy switched completely from custom-made stent-grafts towards the off-the-shelf inner-branch technology (Fig. 4), which allows for the treatment even in narrow aortic anatomies.

Due to the complete occlusion of lumbar and both hypogastric arteries, the patient was regarded to be at tremendous risk for postoperative paraplegia after the thoracoabdominal repair. Our measures to prevent postoperative paraplegia included balanced blood pressure management (mean arterial pressure $>90 \mathrm{mmHg}$ ) and the correction of preoperative anemia to hemoglobin levels $>10 \mathrm{~g} / \mathrm{dL}$. Moreover, at both endovascular stages, the patient received a CSF drain, as he met both high-risk criteria for paraplegia: extensive aortic coverage and the absence of patent lumbar and hypogastric arteries [11]. Finally, the multi-staged approach was performed, which is known to stimulate the spinal collateral network and to reduce the incidence of spinal cord ischemia in comparison to the single stage open or endovascular approach [12].

As a limitation, our case also demonstrates the Achilles' heel of BEVAR: type 111 endoleaks occurred after bridging stent-graft fractures at 3 years. Thus, further evaluation of the durability of BEVAR with the focus on new generation bridging stent-grafts is mandatory to assess endoleak free survival and bridging stent-graft failure [13].

We conclude that the treatment of complex aortic pathologies should be individualized regarding patient comor- bidities and anatomy, while the combination of open and complex endovascular aortic surgery crosses the boundaries imposed by difficult access and insufficient proximal or distal landing zones. Moreover, staged endovascular procedures and the implementation of acknowledged spinal cord protection strategies prevent paraplegia in high-risk patients.

\section{FUNDING}

None.

\section{CONFLICTS OF INTEREST}

Mario Lescan has received educational grants and speaker honoraria from JOTEC Cryolife, Hechingen, Germany. The other author has nothing to disclose.

\section{ORCID}

\author{
Mateja Andic \\ https://orcid.org/0000-0001-7693-8642 \\ Mario Lescan \\ https://orcid.org/0000-0002-3124-4994
}

\section{AUTHOR CONTRIBUTIONS}

Concept and design: all authors. Analysis and interpretation: none. Data collection: none.Writing the article: all authors. Critical revision of the article: all authors. Final approval of the article: all authors. Statistical analysis: none. Obtained funding: none. Overall responsibility: all authors.

\section{REFERENCES}

1) Aftab M, Songdechakraiwut T, Green SY, Zarda S, Price MD, Nalty CC, et al. Contemporary outcomes of open thoracoabdominal aortic aneurysm repair in octogenarians. J Thorac Cardiovasc Surg 2015;149(2 Suppl):S134-S141.

2) Cochennec F, Kobeiter H, Gohel MS, Majewski M, Marzelle J, Desgranges $P$, et al. Impact of intraoperative adverse events during branched and fenestrated aortic stent grafting on postoperative outcome. J Vasc Surg 2014;60:571-578.
3) Rowse JW, Morrow K, Bena JF, Eagleton MJ, Parodi FE, Smolock CJ. lliac conduits remain safe in complex endovascular aortic repair. J Vasc Surg 2019;70:424-431.

4) Khoynezhad A, Donayre CE, Bui H, Kopchok GE, Walot 1, White RA. Risk factors of neurologic deficit after thoracic aortic endografting. Ann Thorac Surg 2007;83:S882-S889; discussion S890-S892.

5) Amabile P, Grisoli D, Giorgi R, Bartoli JM, Piquet P. Incidence and deter- minants of spinal cord ischaemia in stent-graft repair of the thoracic aorta. Eur J Vasc Endovasc Surg 2008;35:455-461.

6) Schlösser FJ, Verhagen $\mathrm{HJ}$, Lin $\mathrm{PH}$, Verhoeven EL, van Herwaarden JA, Moll FL, et al. TEVAR following prior abdominal aortic aneurysm surgery: increased risk of neurological deficit. J Vasc Surg 2009;49:308-314; discussion 314.

7) Tsilimparis N, Dayama A, Perez S, Ricotta JJ 2nd. lliac conduits for 
endovascular repair of aortic pathologies. Eur J Vasc Endovasc Surg 2013;45:443-448; discussion 449.

8) Pyun AJ, Zhang LL, Magee GA, Ziegler KR, Rowe VL, Weaver FA, et al. Use of inner branches during physician-modified endografting for complex abdominal and thoracoabdominal aortic aneurysms. Ann Vasc Surg 2021;76:244253.

9) Abisi S, Zymvragoudakis V, Gkoutzios P, Sallam M, Donati T, Saha P, et al. Early outcomes of Jotec innerbranched endografts in complex en- dovascular aortic aneurysm repair. J Vasc Surg 2021;74:871-879.

10) Mascoli C, Vezzosi M, Koutsoumpelis A, lafrancesco M, Ranasinghe A, Clift $P$, et al. Endovascular repair of acute thoraco-abdominal aortic aneurysms. Eur J Vasc Endovasc Surg 2018;55:92100.

11) Riambau V, Böckler D, Brunkwall J, Cao P, Chiesa R, Coppi G, et al. Editor's choice - management of descending thoracic aorta diseases: clinical practice guidelines of the European Society for Vascular Surgery (ESVS). Eur J
Vasc Endovasc Surg 2017;53:4-52.

12) Etz CD, Zoli S, Mueller CS, Bodian CA, Di Luozzo G, Lazala R, et al. Staged repair significantly reduces paraplegia rate after extensive thoracoabdominal aortic aneurysm repair. J Thorac Cardiovasc Surg 2010;139:1464-1472.

13) Torsello GF, Beropoulis E, Munaò R, Trimarchi S, Torsello GB, Austermann M. Outcomes of bridging stent grafts in fenestrated and branched endovascular aortic repair. J Vasc Surg 2020;72:859-865. 